\title{
ON A THEOREM OF CHERN
}

\author{
BY
}

\author{
J. H. SAMPSON(1)
}

To Professor Chem on his sixtieth birthday

ABSTRACT. A new proof is given for Chern's theorem showing that the Laplace operator for differential forms commutes with decomposition of forms associated with $G$-structures admitting a suitable connection. An analogous result is proved for symmetric tensor fields, and an application is made to determine all harmonic symmetric fields on a compact space of constant negative curvature. Vector-valued forms are al so discussed.

1. Introduction. At the root of the Hodge decomposition of harmonic forms on a Kähler manifold stands the fact that the Laplace operator $\Delta$ commutes with the operator $L: \phi \rightarrow \phi \wedge \Omega$, where $\Omega$ is the fundamental 2-form of the Kähler structure, cf. [6], [11].

In [5] Chern showed that a similar phenomenon holds under broad conditions for general $G$-structures. Here we present a different proof of Chern's theorem, in which the complicated curvature calculations of [5] are replaced by considerations which seem much simpler.

$\$ \$ 2$ and 3 give brief discussion of $G$-structures and connections; in $\$ 4$ a general Laplace operator is defined for vector bundles, and in $\$ 5$ we show that it commutes with certain types of bundle homomorphisms. Our proof of Chern's theorem, based on simple curvature expressions, is given in $\$ 6$. Some applications to well-known facts about the Laplace operator on a Kähler manifold are mentioned in $\$ 7$.

In $\$ 8$ we consider symmetric covariant tensor fields. A Laplace operator $\Delta_{s}$ is defined in a straightforward manner, and it is shown that an analogue of Chern's theorem holds for $\Delta_{s}$. Applications are made in $\$ 9$ to two operators $P$, $Q$ for symmetric fields. These operators commute with $\Delta_{s}$. The harmonic fields for compact spaces of constant curvature are discussed in some detail.

Finally, in $\$ 10$, vector-valued forms are considered briefly. Laplace operators are defined in a natural manner, but the appropriate analogue of Chern's

Received by the editors January 10, 1972.

AMS (MOS) subject classifications (1970). Primary 58A10, 53C20, 53C10; Secondary $22 \mathrm{G} 05$.

Key words and phrases. Laplace operator, $G$-structure, harmonic forms, Killing fields, symmetric fields, orthogonal group.

(1) Partially supported by N. S. F. Grants GP-5980 and GP-8839. 
theorem no longer holds in general. The failure of the analogue is expressed by a certain operator which is exhibited explicitly.

2. Vector bundles. Let $E$ denote an $r$-dimensional vector bundle over a differentiable manifold $M$ (all our ingredients will be assumed $C^{\infty}$ ). A frame $(U, e)$ of $E$ consists of an open $U \subset M$ and an $r$-tuple $e=\left(e_{1}, \cdots, e_{r}\right)$ of $C^{\infty}$ sections over $U$ which span the fibre $E_{a}$ at each point $a$ of $U$. If $\left(V, e^{\prime}\right)$ is another such frame, then in $U \cap V$ we shall have $e^{\prime}=e A\left(e_{a}^{\prime}=e_{\beta} A_{a}^{\beta}\right)$, where $A$ is a matrix-valued function on $U \cap V$, called the transition function for the pair of frames.

$G$ being a Lie group with a faithful representation $\rho$ on $\mathbf{R}^{r}$, a $(G, \rho)$-structure on $E$ (we shall say simply $G$-structure) consists of a covering $\mathfrak{U}$ of $M$ by frames of $E$ such that the transition function of any two overlapping frames in $\mathfrak{U}$ has values in the image $\rho(G)$ in GL $\left(\mathbf{R}^{r}\right)$. A frame of $E$ will be called a $G$-frame if $\mathfrak{U}$, when augmented by the adjunction of this frame, still defines a $G$-structure. Observe that a $G$-structure on $E$ induces a $G$-structure on the dual bundle $E^{*}$, corresponding to the representation ${ }^{t} \rho^{-1}$.

Let $E, E$ be two bundles over $M$ with $G$-structures associated with representations $\rho \tilde{\rho}$. We shall call the $G$-structures coberent if there is given a oneto-one correspondence between $G$-frames of $E$ and $\widetilde{E}$ (over the same open sets) such that for any two pairs of corresponding $G$-frames, say $(U, e),(U, \tilde{e})$ and $\left(V, e^{\prime}\right),\left(V, \tilde{e}^{\prime}\right)$, the transition matrices from $e$ to $e^{\prime}$, resp. $\tilde{e}$ to $\tilde{e}^{\prime}$, at each point of $U \cap V$ correspond to the same element of $G$ in the representations $\rho, \tilde{\rho}$.

If that is so, then the frames $(U, e \otimes \tilde{e})$ of $E \otimes \widetilde{E}$ define a $G$-structure on $E \otimes \widetilde{E}$, and in a natural way it is coherent with the given $G$-structures on $E, \widetilde{E}$; it is associated with the representation $\rho \otimes \tilde{\rho}$ of $G$. In a similar way we can define $G$-structures on exterior powers $\wedge^{p} E$, etc. (cf. $\$ 6$ ).

3. Connections. Let $\Gamma(E)$ denote the module of $C^{\infty}$ sections of the vector bundle $E$ over $M$; and let $T^{*}=T^{*}(M)$ denote the cotangent bundle of $M$. A connection $D$ on $E$ is an R-linear mapping $D: \Gamma(E) \rightarrow \Gamma\left(E \otimes T^{*}\right)$ which is local (i.e. diminishes supports) and satisfies $D(f s)=s \cdot d f+f \cdot D s$ for any $C^{\infty}$ function $f$ and any section $s \in \Gamma(E)$; cf. $[4, \S 2]$. (We omit the symbol $\otimes$ when convenient.)

If $(U, e)$ is a frame of $E$, then $D$ gives on $U$ a connection matrix $L=\left(L_{\beta}^{a}\right)$ of 1 -forms on $U$ defined by $D e_{\beta}=e_{a} L_{\beta}^{a}$. The curvature matrix of the connection $D$ relative to the frame is the matrix of 2 -forms $K=d L+L \wedge L\left(K_{\beta}^{a}=d L{ }_{\beta}^{a}+\right.$ $\left.L_{\gamma}^{a} \wedge L_{\beta}^{\gamma}\right)$.

Now let $E$ have a $G$-structure. We call $D$ a $G$-connection if the matrix $L$ has values in the Lie algebra $g$ of $\rho(G)$ whenever the frame $(U, e)$ is a $G$-frame. 
If that is so, then the curvature matrix $K$ also has values in g. Indeed, if $\xi$ and $\eta$ are tangent vector fields near $a \in U$, then $2 d L(\xi, \eta)=\xi \cdot L(\eta)-\eta \cdot L(\xi)-$ $L([\xi, \eta])$, and this lies in $g$ simply because $g$ is a vector space. And $2 L \wedge L(\xi, \eta)$ $=[L(\xi), L(\eta)]$, which is in $g$ because $g$ is closed under the bracket operation (cf. [3] and 17, Chapter 9] for Kähler connections).

Let $E, \widetilde{E}$ be bundles over $M$ with coherent $G$-structures, as defined above. Let $D, \tilde{D}$ denote $G$-connections on $E$ resp. $\tilde{E}$. We shall call the connections coberent if for any pair of corresponding $G$-frames of $E, \tilde{E}$ the connection matrices have values which correspond under the isomorphism of $\rho(G)$ and $\tilde{\rho}(G)$ given by $\tilde{\rho}^{\circ} \rho^{-1}$. If that is so, then it is easy to verify that the connection $\bar{D}$ on $E \otimes E$ defined by $\bar{D}(s \otimes \tilde{s})=(D s) \otimes \tilde{s}+s \otimes \tilde{D} \tilde{s}$ (cf. [4, p. 80]) is a G-connection for the induced $G$-structure on $E \otimes \tilde{E}$. (In the term $(D s) \otimes \tilde{s}$ it is understood that the factor in $T^{*}$ is to be put on the right.)

4. Laplace operator. We now suppose that $E$ is equipped with a connection $D$ and that $M$ is equipped with a Riemannian metric $g=g_{i j} d x^{i} d x^{j}$. With $D$ and $g$ we now associate a "Laplace operator" $\mathcal{Q}$ on $\Gamma(E)$ as follows: If $s \in \Gamma(E)$, then $D s \in \Gamma\left(E \otimes T^{*}\right)$. Let $D_{0}$ denote the Riemannian connection (i.e. on $\left.T^{*}\right)$. As indicated above, we obtain a connection $\bar{D}$ on $E \otimes T^{*}$ by the rule $\bar{D}(s \omega)=$ $(D s) \omega+s\left(D_{0} \omega\right)$ for any 1 -form $\omega$. We define

$$
\mathcal{L}_{s}=-\operatorname{trace} \bar{D} D s
$$

for $s \in \Gamma(E)$. Relative to a frame $(U, e)$ of $E$ and local coordinates $\left(x^{i}\right)$ in $U$, $\bar{D} D s$ has an expression $s_{i j}^{a} e_{a} \otimes d x^{i} \otimes d x^{j}$, and then $\mathcal{L} s=-\left(g^{i j} s_{i j}^{\alpha}\right) e_{\infty}$, where the $g^{i j}$ are the components of the contravariant metric tensor.

5. G-homomorphisms. Let $E, \tilde{E}$ be vector bundles over $M$ of dimension $r$, $\widetilde{r}$, with coherent $G$-structures associated with representations $\rho, \tilde{\rho}$ of $G$, and with coherent $G$-connections $D, \tilde{D}$. Let $F=\left(f_{\beta}^{a}\right)$ be an $\tilde{r} \times r$ matrix such that $\tilde{\rho}(u) F=F \rho(u)$ for all $u \in G$. From this matrix we obtain a bundle homomorphism, also called $F$, from $E$ to $\tilde{E}$. Namely let $(U, e)$, resp. $(U, \tilde{e})$, be corresponding $G$-frames of $E, \widetilde{E}$. We map $F: e_{\beta} \rightarrow \tilde{e}_{a} f_{\beta}^{a}$, obtaining a homomorphism over $U$. Evidently it does not depend upon the choice of corresponding $G$-frames, and thus we obtain a global homomorphism $F: E \rightarrow \tilde{E}$.

Again let $T^{*}$ carry a Riemannian metric $g$, as in the preceding paragraph. With $g \sim$ and $D$, resp. $\widetilde{D}$, we associate Laplace operators $\mathcal{L}$, resp. $\widetilde{\mathcal{L}}$, on $\Gamma(E)$, resp. $\Gamma(\tilde{E})$. By a direct computation we find that $F \mathscr{\complement}=\widetilde{\mathscr{L}} F$. Apart from the essential assumption of coherence, the chief point involved here is that the bundle homomorphism $F$, relative to corresponding $G$-frames, has constant coefficients. The calculation presents no difficulty and we therefore omit it here. 
6. Differential forms. We now turn to the bundles $E^{p}=\wedge^{p} T^{*}$ of $p$-forms M. We suppose that $T^{*}=E^{1}$ has a given $G$-structure such that $\rho(G) \subset O_{n}, \rho$ denoting a faithful representation of $G$ on $\mathbf{R}^{n}$, where $n=\operatorname{dim} M$. If we take the $G$-frames as orthonormal frames, then of course we obtain a Riemannian metric $g$ on $M$. If $D$ is a $G$-connection on $T^{*}$, and if $D$ has no torsion, then by a classical theorem $D$ coincides with the Riemannian (i.e. Levi-Civita) connection.

By the general rules alluded to in $\$ 2$, the $G$-structure on $E^{1}$ induces a $G$ structure on each $E^{p}$. If $(U, \theta)$ is a frame of $E^{1}$, then it gives rise to a frame $\left(U, \theta^{(p)}\right)$. of $E^{p}$, where $\theta^{(p)}=\left(\theta^{i_{1} \cdots i_{p}}\right)_{i_{1}<\cdots<i_{p}}$ and $\theta^{i_{1} \cdots i_{p}}=\theta^{i_{1}} \wedge \cdots \wedge \theta^{i_{p}}$. If $(U, \theta)$ is a $G$-frame, then $\left(U, \theta^{(p)}\right)$ is a $G$-frame of $E^{p}$.

The connection $D$ defines a connection, also called $D$, on each $E^{p}$. For example, for $p=2, D(\omega \wedge \phi)=(D \omega) \wedge \phi+\omega \wedge(D \phi)$, being induced by the tensor product (it is understood here that the 1 -form arising from $D$ is to be written on the right). If $D$ is a $G$-connection on $E^{1}$, then the extension $D$ is a $G$-connection on $E^{p}$, as is plain.

The Laplace operator $\Delta$ on $p$-forms has the expression

$$
\Delta=\mathfrak{L}+\mathfrak{R}^{(p)}+\mathfrak{S}^{(p)},
$$

where $R^{(p)}, S^{(p)}$ are endomorphisms of $E^{p}$ defined by the Riemannian curvature $[9, \S 26]$. Namely, relative to a frame $(U, \theta)$ for $T^{*}$,

and

$$
R^{(p)}\left(\theta^{i} 1^{\cdots i} p\right)=-\sum_{\nu=1}^{p} \theta^{i}{ }^{\cdots}{ }^{2}{ }^{\cdots i} p_{R_{b}}^{i}, \quad p \geq 1,
$$

$$
\delta^{(p)}\left(\theta^{i 1^{\cdots i} p}\right)=-\sum_{\mu<\nu} \theta^{i{ }^{\cdots} \cdots j_{\mu} \cdots b_{\nu} \cdots{ }^{i} p_{R}{ }^{i} \mu^{i} \nu}, \quad p \geq 2,
$$

where $R_{j b}^{k l}=g^{l q} R_{q j b}^{k}$ is the Riemann curvature operator and $R_{b}^{k}=R_{j b}^{k j}$ is the Ricci tensor (our notation differs slightly from that of de Rham, loc, cit.). In the formula $j_{\mu}$, resp. $b_{\nu}$, signify that $j, b$ appear in the $\mu$ th, resp. $\nu$ th, place. Of course, we have $S^{(p)}=0$ for $p=0,1$; and $R^{(0)}=0$.

We now assume that the Riemannian connection $D$ is a $G$-connection, and that the frame $(U, \theta)$ is a $G$-frame. For each $(k, l)$, the matrix $(i, j) \mapsto R_{j k l}^{i}$ is in the Lie algebra $g$ of $\rho(G)$, as explained in $\S_{3}$. Let $B_{\lambda}=\left(b_{j \lambda}^{i}\right), \lambda=1, \cdots, t$, be a basis for g. Then we may write $R_{j k l}^{i}=\Sigma_{\lambda=1}^{t} a_{\lambda}(k, l) b_{j \lambda}^{i}$ for certain $a_{\lambda}$. Recalling that $g_{i j}=\delta_{i j}$, and using the symmetry properties of the curvature tensor, we obtain the expression $R_{j k l}^{i}=R_{k l}^{i j}=\Sigma_{\lambda, \mu} a_{\lambda \mu} b_{j \lambda}^{i} b_{l \mu}^{k}$, where $a_{\lambda \mu}=a_{\mu \lambda}$. From the vanishing of the torsion tensor we have $R_{j k l}^{i}+R_{l j k}^{i}+R_{k l j}^{i}=0$, from which it follows that the preceding expression can also be written 


$$
R_{k l}^{i j}=\sum a_{\lambda \mu}\left(b_{k \lambda}^{i} b_{l \mu}^{j}-b_{l \lambda}^{i} b_{k \mu}^{j}\right)
$$

and, for the Ricci tensor

$$
R_{b}^{i}=\sum a_{\lambda \mu} b_{j \lambda}^{i} b_{b \mu}^{j}
$$

(recall that the $B_{\lambda}$ are skew-symmetric, since $\rho(G) \subset O_{n}$ ).

Now consider a homomorphism $F$ from the typical fibre $\bigwedge^{p} \mathbf{R}^{n}$ of $E^{p}$ to the typical fibre $\bigwedge^{q} \mathbf{R}^{n}$ of $E^{q}$. Let us assume that $F$ is equivariant with respect to the representations $\Lambda^{p} \rho$, resp. $\Lambda^{q} \rho$. Then (see $\$ 5$ above) it is quickly seen that $F$ induces a bundle homomorphism $F: E^{p} \rightarrow E^{q}$ and an associated homomorphism $F: \Gamma\left(E^{p}\right) \rightarrow \Gamma\left(E^{q}\right)$. Namely, relative to the canonical bases of the typical fibres, $F$ is given by an alternating tensor with certain components $f_{j_{1} \ldots j_{q}}^{i_{1} \ldots i_{p}}$. For our $G$-frame, the bundle map is then given (over $U$ ) by

$$
\theta^{i_{1} \cdots i} p \mapsto f_{j_{1} \cdots j_{q}}^{i_{1} \cdots i_{p}} \theta^{j_{1} \cdots j_{q}} .
$$

If $G$ is connected, as we henceforth assume, the equivariance condition for $F$ is equivalent to the infinitesimal condition

$$
\sum_{\nu=1}^{p} f_{j_{1} \cdots j_{q}}^{i} \cdots b_{\nu} \cdots p_{b_{b}}^{i}{ }_{\nu}=\sum_{\nu=1}^{q} f_{j_{1} \cdots b_{\nu} \cdots j_{q}}^{i_{1} \cdots i_{j}} b_{j}^{b}
$$

for every $B=\left(b_{j}^{i}\right)$ in the Lie algebra g. Let $C=\left(c_{j}^{i}\right)$ be another element of $g$. In the equation above we change $j_{\mu}$ to $k$, multiply by $c_{j \mu}^{k}$ and sum, obtaining

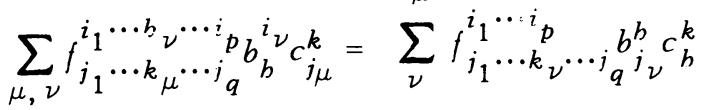

$$
\begin{aligned}
& +\sum_{\mu \nu} f_{j_{1} \cdots h_{\mu} \cdots k_{\nu} \cdots j_{q}}^{i_{1} \cdots b_{j}}\left(b_{j_{\mu}}^{b} c_{\nu}^{k}-b_{j_{\nu}}^{b} c_{j_{\mu}}^{k}\right) .
\end{aligned}
$$

Now in the equivariance equation above change $b_{b}^{i \nu}$ to $c_{k}^{i \mu}$, multiply the new equation by $b_{b}^{i \nu}$ and sum, obtaining

$$
\begin{aligned}
& \sum_{\mu, \nu} f_{j_{1} \cdots k_{\mu} \cdots j_{q}{ }^{i} \cdots b_{b} \cdots p_{b}}^{i} b_{j_{\mu}} c^{k}=\sum_{\nu} f_{j_{1} \cdots j_{q}}^{i_{1} \cdots k_{\nu} \cdots p_{p}} b_{b}^{i} c_{k} c_{k}^{b} \\
& \left.+\sum_{\mu<\nu} f_{j}^{i}{ }_{1}^{i} \cdots b_{q} \cdots p_{\nu} \cdots{ }^{i} p_{(} b_{b}^{i} \mu_{c}{ }^{i} \nu_{k}-b_{b}^{i}{ }^{i} c_{k}^{i} \mu\right) .
\end{aligned}
$$

Thus the right-hand members of the last two equations are equal. From our formulas above for $R_{k l}^{i j}$ and $R_{b}^{k}$, it follows at once that $F\left(\mathscr{R}^{(p)}+\mathfrak{S}^{(p)}\right)=$ $\left(\mathfrak{R}^{(q)}+\delta^{(q)}\right) F$. Indeed one has merely to replace $B$, resp. $C$, by $B_{\lambda}$, résp. $B_{\mu}$, then multiplying by $a_{\lambda \mu}$ and summing.

Finally we observe that the differential operator $\&$ commutes with $F$, as indicated in $\$ 5$. Thus we have proved Chern's theorem: 
Let $F: E^{p} \rightarrow E^{q}$ be an equivariant homomorphism of the bundles of differential forms, in the sense described above. If the structure group $G$ of $T^{*}$ is connected, and if the connection of the G-metric is a G-connection, then the Laplacian $\Delta$ commutes with $F$.

7. Kähler manifolds. We consider exterior forms on a Hermitian manifold $M$. The exterior derivative $d$ and the codifferential $\delta$ split into sums $d=d^{\prime}+d^{\prime \prime}$, resp. $\delta=\delta^{\prime}+\delta^{\prime \prime}$, of bidegrees $(1,0),(0,1),(-1,0),(0,-1)$ respectively (cf. [11]). Now $\Delta=d \delta+\delta d=\left(d^{\prime} \delta^{\prime}+\delta^{\prime} d^{\prime}\right)+\left(d^{\prime \prime} \delta^{\prime \prime}+\delta^{\prime \prime} d^{\prime \prime}\right)+\left(d^{\prime} \delta^{\prime \prime}+\delta^{\prime \prime} d^{\prime}\right)+\left(d^{\prime \prime} \delta^{\prime}+\delta^{\prime} d^{\prime \prime}\right)$. The last two terms are of bidegree $(1,-1)$, resp. $(-1,1)$. If the Hermitian metric is in fact a Kähler metric, then the metric connection is compatible with the complex structure, and the Laplace operator on $p$-forms is compatible with the decomposition of forms according to complex type. Hence the last two terms in the expression above for $\Delta$ are zero. Call the first two terms $\Delta^{\prime}$, resp. $\Delta^{\prime \prime}$. Thus our equation is $\Delta=\Delta^{\prime}+\Delta^{\prime \prime}$. Since $\delta^{\prime}$ is the formal adjoint of $d^{\prime}$ and $\delta^{\prime \prime}$ is the formal adjoint of $d^{\prime \prime}$, the operators $\Delta^{\prime}$ and $\Delta^{\prime \prime}$ are real. By degree considerations we have $\overline{\Delta^{\prime} \phi}=\Delta^{\prime \prime} \bar{\phi}$ (bar denotes complex conjugate); there follows $\Delta=2 \Delta^{\prime}=$ $2 \Delta^{\prime \prime}$ (see [11, Chapter II; Théorème 2]).

If $\Omega=(i / 2) g_{a \bar{\beta}} d z^{a} d \bar{z}^{\beta}$ denotes the fundamental 2-torm of the Kähler structure on $M$, then we obtain a bundle homomorphism $L: E^{p} \rightarrow E^{p+2}$ by $L: \phi \rightarrow$ $\phi \wedge \Omega$. This operator satisfies the requirements of the theorem of $\$ 6$ and therefore commutes with $\Delta$. This fact leads to Hodge's canonical decomposition of a harmonic form into a sum of harmonic forms $L^{r} \phi_{r}$, where the $\phi_{r}$ are primitive. See [11, Chapter 2, Theorem 2] and [6, \$43]. In a remarkable observation, Serre $[10$, Chapter IV, $\$ 4]$ has shown that Hodge's decomposition theorem can be connected with the representation theory of $8 l_{2}$ (cf. $\$ 9$ below).

8. Symmetric fields. $M^{n}$ again being a Riemannian manifold, we now consider the bundles $S^{p}$ of covariant symmetric tensors of rank $p$. For brevity we shall call sections of $S^{p} p$-fields. The symmetrization operator, call it $s_{p}$, gives a projection of the full covariant tensor bundle $T^{0, p}(M)$ onto $S^{p}$.

As before, we assume that $T^{*}(M)=S^{1}$ has a $G$-structure which is a reduction of the $O_{n}$-structure given by the metric. Then on each $S^{p}$ we obtain a $G$-structure by tensor products. If $(U, \theta)$ is a frame for $S^{1}, \theta=\left(\theta^{1}, \ldots, \theta^{n}\right)$, then the sections $\theta_{s}^{i_{1} \cdots i_{p}}=s_{p}\left(\theta^{i_{1}} \otimes \cdots \otimes \theta^{i} p\right), i_{1} \leq \cdots \leq i_{p}$, form a frame for $S^{p}$ over $U$. If $(U, \theta)$ is a $G$-frame, then this associated frame of $S^{p}$ is a $G$-frame. We install upon $S^{p}$ the fibre metric for which those sections are orthonormal at each point of $U$. We write $\left\langle\phi, \phi^{\prime}\right\rangle_{\dot{a}}$ for the inner product of two elements of the fibre $S_{a}^{p}$.

For a $p$-field $\phi$ we define a derivative by $D_{s} \phi=(p+1)_{s_{p+1}}(D \phi)$, where $D$ 
denotes covariant differentiation relative to the Riemannian connection. Relative to a frame the components are given by the formula

$$
\left(D_{s} \phi\right)_{i_{1} \cdots i_{p}}=\sum_{\nu=1}^{p+1} \phi_{i_{1} \ldots \hat{\nu} \cdots i_{p+1}, i_{\nu}}
$$

For $p$-fields $\phi, \phi^{\prime}$ we introduce the inner product

$$
\left(\phi, \phi^{\prime}\right)=\int_{M}\left(\phi, \phi^{\prime}\right\rangle_{a} d V_{a}
$$

where $d V_{\dot{a}}=\sqrt{g}\left|\theta^{1} \wedge \ldots \wedge \theta^{n}\right|$ is the volume element at the point $a$.

The formal adjoint $D_{s}^{*}$ of $D_{s}$ is the operator characterized by the equation $\left(D_{s} \phi, \psi\right)=\left(\phi, D_{s}^{*} \psi\right)$ for an arbitrary $p$-field $\phi$ and an arbitrary $(p+1)$-field $\psi$ whose common support is compact. It is easily shown that for a $p$-field $\phi$ the components of $D_{s}^{*} \phi$ are given by the formula

$$
\left(D_{s}^{*} \phi\right)_{i_{1} \cdots i_{p-1}}=-g^{j k} \phi_{i_{1} \cdots i_{p-1}} \text {. }
$$

Of course we define $D_{s}^{*}=0$ for 0 -fields.

In analogy with the theory of harmonic integrals, we introduce the Laplace operator $\Delta_{s}=D_{s}^{*} D_{s}-D_{s} D_{s}^{*}$. It can be shown that for $p$-fields we have $\Delta_{s}=$ $\mathcal{L}+R^{\prime}(p)+\mathcal{S}^{\prime(p)}$, with the following formulas for the operation on the frame elements introduced above:

$$
\begin{aligned}
& \mathcal{L}\left(\theta_{s}^{i} 1^{\cdots i} p\right)=-g^{j k} \theta_{s}^{i{ }^{1} \cdots{ }^{i} p}, j k, \\
& \mathbb{R}^{\prime(p)}\left(\theta_{s}^{i}{ }^{\cdots}{ }^{\cdots i} p\right)=\sum_{\nu=1}^{p} \theta_{s}^{i 1 \cdots b} \nu^{\cdots i} p_{R_{b}}^{i}, \quad p \geq 1, \\
& \mathcal{S}^{\prime(p)}\left(\theta_{s}^{i 1}{ }^{\cdots i} p\right)=\sum_{\mu<\nu} \theta_{s}^{i_{1} \cdots j_{\mu} \cdots b_{\nu} \cdots{ }_{p}} \bar{R}_{i h}^{i} \mu^{i} \nu, \quad p \geq 2 .
\end{aligned}
$$

Again $j_{\mu}, b_{\nu}$ indicate the positions of the indices $j, b$; and we have $R^{\prime(0)}=0$ and $\mathcal{S}^{\prime(p)}=0$ for $p=0,1$.

Here we have the new curvature operator $\bar{R}_{k l}^{j b}=g^{b q} R_{k q l}^{j}+g^{j q} R_{k q l}^{b}$. Let us assume again that the frame $(U, \theta)$ is a $G$-frame. Referring back to our expression for the curvature in $\$ 6$, we obtain for $\bar{R}$ the expression

$$
\bar{R}_{k l}^{j b}=\sum a_{\lambda \mu}\left(b_{k \lambda}^{j} b_{l \mu}^{b}+b_{k \lambda}^{b} b_{l \mu}^{j}\right)
$$

Observe that $R_{l}^{j}=\bar{R}_{b l}^{j b}$, as follows from the skew-symmetry of the matrices $\left(b_{j \lambda}^{i}\right)$.

Let $F^{\prime}$ denote an equivariant homomorphism, relative to the $G$-action, from the typical fibre of $S^{p}$ to the typical fibre of $S^{q}$. Then $F^{\prime}$ induces a bundle homomorphism $F^{\prime}: S^{p} \rightarrow S^{q}$ (see $\S 5$ ). For the $G$-frame $(U, \theta), F^{\prime}$ is given by a symmetric tensor: 


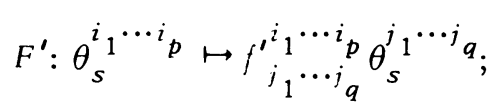

the components $f^{\prime}$ are constant and independent of the choice of $G$-frame, and they satisfy symmetry relations similar to those given for $F$ in $\$ 6$. From the similarity of the expressions for $\Delta$, resp. $\Delta_{s}$, it is clear that, with only minor modifications, the argument of $\$ 6$ can be applied to $\Delta_{s}$, and we obtain the following theorem:

Let $F^{\prime}: S^{p} \rightarrow S^{q}$ be an equivariant bundle mapping, as described above. If the structure group $G$ is connected, and if the Riemannian connection is a G-connection, then $\Delta_{s}$ commutes with $F^{\prime}$.

9. Harmonic fields. We shall continue with some observations concerning barmonic $p$-fields, i.e. fields $\phi$ satisfying $\Delta_{s} \phi=0$. To simplify statements, we shall assume that $M$ is compact.

The operator $\Delta_{s}$ is related to a variational problem, as follows: Define the "energy" of a $p$-field $\phi$ by $E(\phi)=1 / 2\left(D_{s} \phi, D_{s} \phi\right)-1 / 2\left(D_{s}^{*} \phi, D_{s}^{*} \phi\right)=1 / 2\left(\phi, \Delta_{s} \phi\right)$. For a variation $\delta \phi$ of $\phi$ we obtain $\delta E(\phi)=\left(\delta \phi, \Delta_{s} \phi\right)$. Hence $\Delta_{s} \phi=0$ is the condition for a free extremal of $E$. If we impose the constraint $(\phi, \phi)=1$, say, then according to the theory of Lagrange multipliers, the extremal condition becomes $\Delta_{s} \phi=c \phi(c=$ const $)$. Of course the energy functional $E(\phi)$ is not always positive.

Consider now a 1 -field $\phi$ on $M$, i.e. a Pfaffian form. If $D_{s} \phi=0$, then necessarily $D_{s}^{*} \phi=0$, as is readily seen, and so $\Delta_{s} \phi=0$. Now $D_{s} \phi=0$ is none other than Killing's equation, and so Killing fields are harmonic fields (see [2]). A $p$-field whose covariant derivative vanishes is clearly harmonic, the metric tensor $g$ being the most important example.

In general, geometric interpretations of the spaces of harmonic $p$-fields are not known. Presently we shall take up in detail the case of constant curvature $K$. Then the curvature operator has components $\bar{R}_{k l}^{j b}=K\left(2 g^{j b} g_{k l}-\delta_{k}^{j} \delta_{l}^{b}-\delta_{l}^{j} \delta_{k}^{b}\right)$, and the Ricci tensor is $R_{l}^{j}=K(1-n) \delta_{l}^{j}$. The operators $R^{\prime(p)}$ and $\mathcal{S}^{\prime(p)}$ then take on a particularly simple form.

Before imposing the curvature restriction, however, we shall develop a very useful formalism involving the metric tensor.

Let us denote the symmetric product by $V$. Thus e.g. for 1 -fields $\xi$, $\eta$ we have $\xi \vee \eta=1 / 2(\xi \otimes \eta+\eta \otimes \xi)$. Define operators $P, Q$ for $p$-fields by

$$
\begin{aligned}
P \phi & =-1 / 2(p+1)(p+2) \cdot \phi \vee g ; \\
Q & =-(p+1)^{-1}(p+2)^{-1} P^{*} \text { for } p \geq 2, \\
Q & =0 \text { for } p=0,1 .
\end{aligned}
$$


Here $P^{*}$ denotes the adjoint of $P$, and $g$ is the metric tensor. For components we have the formulas

$$
\begin{aligned}
& (P \phi)_{i_{1} \cdots i_{p+2}}=-\sum_{\mu<\nu} \phi_{i_{1} \cdots \hat{\mu} \cdots \hat{\nu} \cdots i_{p+2}} \cdot g_{i_{\mu}{ }^{i} \nu}, \quad p \geq 2 ; \\
& (Q \phi)_{i_{1} \cdots i_{p-2}}=1 / 2 \phi_{i_{1} \cdots i_{p-2^{j k}}} \cdot g^{j k}, \quad p \geq 2 .
\end{aligned}
$$

We have the commutation relations $D_{s} P-P D_{s}=0, D_{s} Q-Q D_{s}=D_{s}^{*}$, and $P Q-$ $Q P=n / 2+p$ (for $p$-fields). These hold for $p \geq 0$. Write $W$ for the latter commutator. Then $[P, Q]=W,[W, P]=2 P,[W, Q]=-2 Q$. Therefore the typical fibre of the bundle $S=\Sigma_{p=0}^{\infty} S^{p}$ becomes an $\delta L_{2}(R)$-module (cf. Serre [10, Chapitre 4]).

It is readily verified that $P$ and $Q$ are equivariant bundle maps for the $O_{n}$ structure of $T^{*}$, in the sense understood in $\$ 8$. We may therefore apply the theorem of $\$ 8$ to conclude that $\Delta_{s}$ commutes with $P$ and $Q$. This may also be seen from the commutation rules above. The situation is in striking analogy with the Kähler case, but does not lead to parallel conclusions because of the infinite dimensionality of our representation of $z \Upsilon_{2}$.

We have subbundles $\operatorname{Ker} Q \subset \operatorname{Ker} Q^{2} \subset \operatorname{Ker} Q^{3} \subset$ etc., from which we obtain the orthogonal decomposition $S^{p}=S_{0}^{p}+\cdots+S_{\langle p\rangle}^{p}(\langle p\rangle=p / 2$ for even $p$ and $(p-1) / 2$ for odd $p)$, where $S_{k}^{p}$ is the orthogonal complement of the bundle $S^{p} \cap$ $\operatorname{Ker} Q^{k}$ in $S^{p} \cap \operatorname{Ker} Q^{k+1}$. The subspace $S_{0, a}^{p}$ of the fibre $S_{a}^{p}$ is irreducible for the orthogonal group of $T_{a}^{*}$ (see $[12$, Chapter $5, \S 7]$ ). The subspaces $S_{k, a}^{p}$ are also irreducible, as will presently be evident.

The operators $P Q$ and $Q P$ are selfadjoint in each fibre $S_{a}^{p}$. The commutator $\left[P, Q^{k}\right]$ is a multiple of $Q^{k-1}$ on $p$-fields, from which it is easily seen that $Q$ maps $S_{k}^{p}$ into $S_{k-1}^{p-2}(k \geq 1)$, and that each subbundle $S_{k}^{p}$ is stable for $P Q$ and $Q P$. On $S_{k}^{p}$ we have

$$
P Q\left|S_{k}^{p}=-k(n / 2+p-k-1), \quad Q P\right| S_{k}^{p}=-(k+1)(n / 2+p-k) .
$$

This is easily established by induction on $k$. For let $\phi \in S_{k}^{p}, k \geq 1$, and write $P Q \phi=\psi$. Then $Q P Q \phi=Q \psi$; and $Q \phi, Q \psi$ are in $S_{k-1}^{p-2}$. By induction assumption, $Q P Q \phi=c Q \phi$, for a certain factor $c$, whence $Q(c \phi-\psi)=0$. But $c \phi-\psi$ is in $S_{k}^{p}$ and thus is orthogonal to $\operatorname{Ker} Q$, whence $c \phi=\psi$, and so $P Q \phi=c \phi$. The value of $c$ is quickly found inductively with use of the relation $Q P=P Q-$ $(n / 2+p)$.

As just pointed out, $Q$ maps $S_{k}^{p}$ injectively into $S_{k-1}^{p-2}$ for $k>0$. We see now that $P$ is injective. Indeed, if $\phi \in S_{k}^{p}$ and $P \phi=0$, then $Q P \phi=0$, and so $P Q \phi=(n / 2+p) \phi$. Comparing with the result above for $P Q \mid S_{k}^{p}$, we obtain $\phi=0$. 
The harmonic elements in $\Gamma\left(S^{p}\right)$ form a finite-dimensional vector space $H^{p}$, for compact $M$, as follows from standard elliptic theory applied to the strongly elliptic system $\Delta_{s} \phi=0$; and the equation $\Delta_{s} \phi=\psi$ for $C^{\infty}$ fields has a solution if and only if $\psi$ is orthogonal to all elements of $H^{p}$. See for example [1], [8]. Since $\Delta_{s}$ commutes with $P$ and $Q$, the space $H^{p}$ splits into an orthogonal sum $H_{0}^{p}+\cdots+H_{\langle p\rangle}^{p}$, with $H_{k}^{p} \subset \Gamma\left(S_{k}^{p}\right)$.

For a section $\phi$ of $S_{0}^{p}$ we have $D_{s}^{*} \phi=-Q D_{s} \phi$, and so $\phi$ is harmonic if $D_{s} \phi=0$. We observe that the energy functional $E(\phi)$ is $\geq 0$ for $p=1$ and $n \leq 4$. In these special cases it follows that a global harmonic 1 -field $\phi$ satisfies $D_{s} \phi=D_{s}^{*} \phi=0$ on a compact manifold.

By linear algebra, $P$ maps onto the orthogonal complement of $\operatorname{Ker} P^{*}=$ Ker $Q$, and therefore we find that $P$ maps $S_{k}^{p}$ isomorphically upon $S_{k+1}^{p+2}$ $(k=0,1, \cdots,\langle p\rangle)$. As $P$ commutes with $\Delta_{s}, P$ maps $H_{k}^{p}$ isomorphically onto $H_{k+1}^{p+2}$. Thus for harmonic fields it suffices to consider the bundles $S_{0}^{p}$. Since $P$ commutes with $D_{s}$, if $\phi \in \Gamma\left(S_{k}^{p}\right)$ satisfies $D_{s} \phi=0$, then by reducing via $P$ to $k=0$, we see that $\Delta_{s} \phi=0$, and so $D_{s} D_{s}^{*} \phi=0$. For the compact manifold $M$ it follows that $D_{s}^{*} \phi=0$ also.

Let $\phi \in \Gamma\left(S_{0}^{p}\right)$ and $\psi \in \Gamma\left(S_{0}^{q}\right)$. If $\phi, \psi$ satisfy the orthogonality relation expressed in terms of components by

$$
\phi_{i_{1} \cdots i_{p-1}} \psi_{j_{1} \cdots j_{q-1}} g^{k l}=0
$$

then it is not hard to see that $\phi \vee \psi$ is a section of $S_{0}^{p+q}$. Since, moreover,

$$
D_{s}(\phi \vee \psi)=\frac{p+q+1}{p+1}\left(D_{s} \phi\right) \vee \psi+\frac{p+q+1}{q+1} \phi \vee\left(D_{s} \psi\right)
$$

it follows that $D_{s}(\phi \vee \psi)=0$, hence $\phi \vee \psi$ harmonic, whenever $D_{s} \phi=D_{s} \psi=0$. For example, if $\phi^{1}, \cdots, \phi^{p}$ are orthonormal covariant Killing fields, then $\phi^{1} \vee \ldots \vee \phi^{p}$ is a harmonic section of $S_{0}^{p}$.

Let us note in passing that the fibre dimension of $S^{p}$ is

$$
\underline{d}_{p}=\sum_{m=1}^{\min (n, p)}\left(\begin{array}{l}
n \\
m
\end{array}\right)\left(\begin{array}{l}
p-1 \\
m-1
\end{array}\right) \text {. }
$$

With the foregoing observations concerning $P: S^{p} \rightarrow S^{p+2}$ in mind, we see that $S_{0}^{p}$ has fibre dimension $d_{p}-d_{p-2}(p \geq 2)$. For example, for $n=2$ this dimension is 2 ; and for $n=3$ it is $2 p+1$.

For $n=2$ the typical fibres of $S_{0}^{p}(p \geq 2)$ are irreducible $O_{2}$-modules (of dimension 2), hence are isomorphic. This means that for any orthonormal basis $b$ of the typical fibre of $S_{0}^{p}$, there is an orthonormal basis $b^{\prime}$ of the typical fibre of $S_{0}^{\dot{q}}(p, q \geq 2)$ for which the $\mathrm{O}_{2}$-action is represented by the same matrices. Clearly $b^{\prime}$ is uniquely determined by $b$, and we may conclude that the bundle,s 
$S_{0}^{p}(p \geq 2)$ are all equivariantly isomorphic. Hence for $n=2$ only the bundles $S_{0}^{1}, S_{0}^{2}$ are of importance in the present connection.

Let us now turn to the case of constant curvature, for which the tensor $\vec{R}$ has the form given earlier. It is readily shown that the following formulas result for a $p$-field $\phi$ :

$$
R^{\prime(p)} \phi=-p K(n-1) \phi, \quad S^{\prime(p)} \phi=-p(p-1) K \phi-4 K P Q \phi .
$$

Thus for a section $\phi$ of $S_{0}^{p}$ the harmonic equation reduces to $\mathcal{L} \phi=p K(n+p-2) \phi$

Suppose that $K \leq 0$. Then for a harmonic section $\phi$ of $S_{0}^{p}(p>0), \mathfrak{Q}=$ $-r \phi$, with $r \geq 0$. Since $\mathcal{L}$ is a positive operator, and $-r$ is a negative operator, there follows $(£ \phi, \phi)=r(\phi, \phi)=0$. If $K<0$, we find that $\phi=0$. Hence, for a compact manifold of constant negative curvature the only harmonic nonzero $p$ fields are those of the form $g \vee \cdots \vee g \times$ const.

If $K=0$, then we have just $(£ \phi, \phi)=0$. But $(£ \phi, \phi)=(D \phi, D \phi)$, and so $D \phi=0$. Thus $(\mathcal{\phi}, \phi)=0$, and in particular $\mathscr{L} \phi=0$ if and only if $\phi$ is a uniform field (i.e. has covariant derivative zero). (The argument here is substantially that of Bochner-cf. [2]).

10. Vector-valued forms. Computations similar to the foregoing can be made for other tensor bundles over $M^{n}$. Here we shall take up rather summarily the bundles of vector-valued $p$-forms $\hat{E}^{p}=E^{p} \otimes T$ and of vector-valued $p$-fields $\hat{S}^{p}=$ $S^{p} \otimes T$, where $T$ denotes the tangent bundle of $M$. These bundles are of interest in connection with deformation theory.

We again suppose that there is given a $G$-structure on $T^{*}$ which is a reduction of a smooth $O_{n}$-structure. If $\left(U,\left(\theta^{i}\right)\right)$ is a frame of $T^{*}$, and if $\left(U,\left(\theta_{i}\right)\right)$ is the dual frame of $T$, then the system $\left(U,\left(\theta_{k}^{i_{1} \cdots i_{p}}\right)\right)_{i_{1}<\cdots<i_{p}}$ is a frame of $\hat{E}^{p}$ over $U$, where $\theta_{k}^{i_{1} \ldots i_{p}}=\theta^{i} 1 \wedge \ldots \wedge \theta^{i p} \otimes \theta_{k}$. If $\left(U,\left(\theta^{i}\right)\right)$ is a $G$-frame, then we declare this associated frame of $\hat{E}^{p}$ to be a $G$-frame; and in this manner we obtain a $G$-structure on $\hat{E}^{p}$.

Further we introduce on $\hat{E}_{a}^{p}$ the fibre metric $(,\rangle_{a}$ for which these elements form an orthonormal basis. In particular, we have once more the Riemannian metric $g=g_{i j} \theta^{i} \theta^{j}$ on $T$, and $g_{i j}=\delta_{i j}$ for a $G$-frame. By means of the metrics we obtain a global inner product

as in $\$ 6$.

$$
\left(\phi, \phi^{\prime}\right)=\int_{M}\left\langle\phi, \phi^{\prime}\right\rangle_{a} d V_{a}
$$

For a section $\phi$ of $\hat{E}^{p}$ we define a derived form $d \phi=(p+1) A_{p+1} D \phi$, where $D$ is the covariant derivative and $A_{p+1}$ is the alternation operator on the "covariant indices". If $\phi$ has components $\phi_{i_{1} \ldots i_{p}}^{k}$ relative to a frame $(U, \theta)$ of $T^{*}$, then $d \phi$ has components 


$$
(d \phi)_{i_{1} \cdots i_{p+1}}^{k}=\sum(-1)^{\nu-1} \phi_{i_{1}}^{k} \ldots \hat{\nu} \ldots i_{p+1}, i_{\nu}
$$

The formal adjoint $d^{*}$ of $d$ is given, for a section $\phi$ of $\hat{E}^{p}$, by

$$
\left(d^{*} \phi\right)_{i_{1} \cdots i_{p-1}}^{k}=-\phi_{b i_{1} \cdots i_{p-1}, l^{g}}^{k},
$$

where as usual the $g^{b l}$ are the components of the metric tensor $g$ relative to the frame in use. For $p=0$ we define $d^{*} \phi=0$.

The corresponding Laplace operator $\Delta=d d^{*}+d^{*} d$ for sections of $\hat{E}^{p}$ has the expression $\Delta=\mathfrak{L}+\mathfrak{R}^{(p)}+\mathcal{S}^{(p)}+\mathcal{J}^{(p)}$, where $\mathcal{L}$ is as in $\S 4$ and $\mathfrak{R}^{(p)}, \mathcal{S}^{(p)}$ are quite as in $\$ 6 . \mathcal{T}^{(p)}$ is the operator given on the frame $\left(U,\left(\theta_{k}^{i_{1} \ldots i_{p}}\right)\right)_{i_{1}<\cdots<i_{p}}$ by

$$
\mathcal{T}^{(p)} \theta_{k}^{i_{1} \cdots{ }^{\prime} p}=\sum_{\nu=1}^{p} \theta_{l}^{i_{1} \cdots h_{\nu} \cdots{ }^{i}} \hat{R}_{k b}^{l i} \quad(p>0),
$$

where $\hat{R}_{k b}^{l i}=g^{i m} R_{k m b}^{l}$.

Now let $F$ be an equivariant linear mapping $\wedge^{p} \mathbf{R}^{n} \otimes \mathbf{R}^{n} \rightarrow \wedge^{q} \mathbf{R}^{n} \otimes \mathbf{R}^{n}$. Relative to the canonical bases $F$ is given by a system of components $f_{j_{1} \ldots j_{q}}^{i_{1} \ldots i_{p} l}$ which are skew-symmetric in the $i$ 's, resp. the $j$ 's. As in $\$ 6, F$ gives a bundle homomorphism $F: \hat{E}^{p} \rightarrow \hat{E}^{q}$ which, for the $G$-frames defined above, is given by

$$
F: \theta_{k}^{i_{1} \cdots \cdots_{p}} \mapsto f_{i_{1} \cdots j_{q}{ }^{i} \cdots i_{p}^{l}}^{l} \theta_{l}^{j} \cdots{ }^{j} q
$$

If $G$ is connected, then the equivariance condition reduces to the following relation, for all elements $\left(b_{j}^{i}\right)$ in the Lie algebra of $G$ :

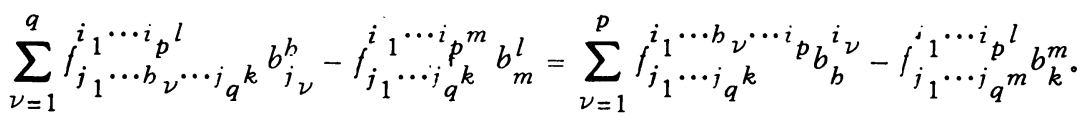

By operating upon this identity as in $\$ 6$, we can determine the commutator $\Delta F-F \Delta$. It is found that this operator is expressed in terms of components by

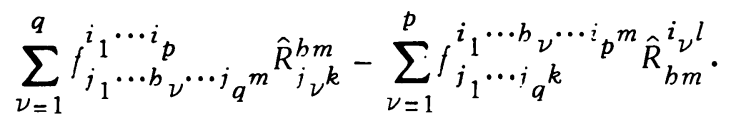

For certain $F$ this commutator vanishes. For example, if our $G$-structure is a Kähler structure with fundamental 2-form $\omega$, and if $F$ denotes the operation $F \phi=\omega \wedge \phi$, then it can be shown by direct computation that $\Delta F=F \Delta$. Thus we can conclude that a Hodge decomposition holds for harmonic vector-valued forms on a Kähler manifold. 
The situation is analogous for vector-valued $p$-fields, i.e. sections of the bundle $\hat{S}^{p}$. A Laplace operator $\Delta_{s}$ is defined as in $\$ 8$. For an equivariant bundle map $F: \hat{S}^{p} \rightarrow \hat{S}^{q}$ of the sort considered above (but with symmetric components $f_{j_{1} \ldots j_{q}}^{i_{1} \ldots i_{p} l}$ in the $i$ 's, resp. $j$ 's, rather than the skew-symmetric ones pertaining to the $\hat{E}^{p}$ ), we find that $\Delta_{s} F-F \Delta_{s}$ is given by the same expression as that just written for the case of exterior forms.

We can define operators $P, Q$ for sections of $\hat{S}^{p}$, just as in $\$ 9$. For these operators the commutator vanishes, as is fairly easily seen.

\section{REFERENCES}

1. P. Bidal and G. de Rham, Les formes différentielles harmoniques, Comment. Math. Helv. 19 (1946), 1-49. MR 8, 93.

2. S. Bochner, Vector fields and Ricci curvature, Bull. Amer. Math. Soc. 52 (1946), 776-797. MR 8, 230.

3. —- Curvature in Hermitian metric, Bull. Amer. Math. Soc. 53 (1947), 179-195. MR 8, 490 .

4. R. Bott and S.-S. Chern, Hermitian vector bundles and the equidistribution of their holomorphic sections, Acta Math. 114 (1965), 71-112. MR 32, \#3070.

5. S. S. Chern, On a generalization of Kähler geometry. Algebraic geometry and topology (A Sympos. in Honor of S. Lefschetz), Princeton Univ. Press, Princeton, N.J., 1957, pp. 103-121. MR 19, 314.

6. W. V. D. Hodge, The theory and application of harmonic integrals, Cambridge Univ. Press, Cambridge, 1941. MR 2, 296.

7. S. Kobayashi and K. Nomizu, Foundations of differential geometry. Vol. II, Interscience Tracts in Pure and Appl. Math., no. 15, vol. II, Interscience, New York, 1969. MR 38 \#6501.

8. R. Palais et al., Seminar on the Atiyah-Singer index theorem, Ann. of Math. Studies, no. 57, Princeton Univ. Press, Princeton, N.J., 1965. MR 33 \#6649.

9. G. de Rham, Variétés différentiables, Actualités Sci. Indust., no. 1222, Hermann, Paris, 1955. MR 16, 957.

10. J.-P. Serre, Algèbres de Lie semi-simples complexes, Benjamin, New York, 1966. MR 35 \#6721.

11. A. Weil, Variétés Kählériennes, Hermann, Paris, 1958.

12. H. Weyl, The classical groups, Princeton Univ. Press, Princeton, N. J., 1939. MR 1, 42 .

DEPARTMENT OF MATHEMATICS, THE JOHNS HOPKINS UNIVERSITY, BALTIMORE, MARYLAND 21218 